arthritis, extra-articular involvement, treatment (during paediatric follow-up and at the time of the first evaluation) and disease activity (using JADAS10-CRP and DAS28-CRP; table 1). Finally, to assess the efficacy of this transition-model, we compared the rate of losses at follow-up with those occurred until 2016, when transition was performed only with a Clinical Report sent by paediatrician.

Table 1 Cutoff of disease activity: JADAS10* and DAS28-CRP**

\begin{tabular}{|l|l|}
\hline & \multicolumn{1}{|c|}{ Cutoff } \\
\hline JADAS10 (for Oligoarthritis) & \\
\hline Inactive disease & $\leq 1$ \\
\hline Low activity & $\leq 1.5$ \\
\hline Moderate activity & $1.51-4$ \\
\hline High activity & $>4$ \\
\hline JADAS10 (for Polyarthritis) & \\
\hline Inactive disease & $\leq 1$ \\
\hline Low activity & $\leq 2.5$ \\
\hline Moderate activity & $2.51-8.5$ \\
\hline High activity & $>8.5$ \\
\hline DAS28-CRP & \\
\hline Inactive disease & $\leq 2.6$ \\
\hline Low activity & $>2.6-3.2$ \\
\hline Moderate activity & $>3.2-5.1$ \\
\hline High activity & $>5.1$ \\
\hline *Modified from Consolaro A. et al., Ann Pediatr Rheum 2014 \\
$*$ Modified from Prevoo ML et al., Arthritis Rheum 1995 \\
\hline
\end{tabular}

Figure 1

Results: Between May 2016 and May 2018, 28 patients were enrolled (6 male, 22 female); mean age at diagnosis was 8.1 years (range 1-6 years); mean age at transition was 18.5 years (range 17-21 years). Twenty patients had a diagnosis of JIA, 3 of Undifferentiated Connective Tissue Disease, 1 Dermatomyositis, 1 Systemic Lupus Erythematosus, 1 Takayasu Arteritis, 1 Morphea, 1 Raynaud's phenomenon.

Among JIA, $6(30 \%)$ were Persistent Oligoarthritis, 4 (20\%) Extended Oligoarthritis, $8(40 \%)$ Polyarthritis (RF negative), 1 (5\%) Psoriatic Arthritis, $1(5 \%)$ Enthesitis Related Arthritis. Five patients $(25 \%)$ had history of uveitis. Treatment during the paediatric follow-up period are summarized in table 2. At the time of first evaluation in adult department, 2 patients $(10 \%)$ were on treatment with systemic steroids $(5 \mathrm{mg} /$ day $), 6(30 \%)$ with methotrexate, $2(10 \%)$ sulfasalazine, $1(5 \%)$ with hydroxychloroquine, 1 $(5 \%)$ leflunomide; 10 patients $(50 \%)$ were on treatment with bDMARDs $(6$ adalimumab, 3 etanercept, 1 infliximab). Mean JADAS10-CRP was 3.8 (range $0-17$, SD 5.8) as mean DAS28-CRP was 1.65 (range 0.96-3.44, SD 0.72). In 7 patients, disease activity assessed with JADAS10 was moderate-high as it resulted low-absent using DAS28-CRP. The rate of patients classified as having moderate-high activity was significantly different using the two index (35\% using JADAS10 vs $5 \%$ using DAS28-CRP, pvalue 0.044)

Until 2016, 6 of $44(11 \%)$ patients were lost to follow-up while in the period between May 2016 and May 2018 no patients were lost $(11 \%$ vs 0 ; pvalue 0.042 )

Conclusion: Disease activity seems to be underestimated using DAS28CRP as compared with JADAS10-CRP. This could be due to the more frequent involvement of ankles and feet (that are not included in DAS28CRP) in JIA. The adherence to follow up was greater in patients whose transition was performed in presence of the two Specialists.
Abstract AB0955 Table 1. Cutoff of disease activity: JADAS10* and DAS28-CRP**

Table 1 Cutoff of disease activity: JADAS10* and DAS28-CRP**

\begin{tabular}{|l|l|}
\hline & \multicolumn{1}{|c|}{ Cutoff } \\
\hline JADAS10 (for Oligoarthritis) & \\
\hline Inactive disease & $\leq 1$ \\
\hline Low activity & $\leq 1.5$ \\
\hline Moderate activity & $1.51-4$ \\
\hline High activity & $>4$ \\
\hline JADAS10 (for Polyarthritis) & \\
\hline Inactive disease & $\leq 1$ \\
\hline Low activity & $\leq 2.5$ \\
\hline Moderate activity & $2.51-8.5$ \\
\hline High activity & $>8.5$ \\
\hline DAS28-CRP & \\
\hline Inactive disease & $\leq 2.6$ \\
\hline Low activity & $>2.6-3.2$ \\
\hline Moderate activity & $>3.2-5.1$ \\
\hline High activity & $>5.1$ \\
\hline *Modified from Consolaro A. et al., Ann Pediatr Rheum 2014 \\
$* *$ Modified from Prevoo ML et al., Arthritis Rheum 1995 \\
\hline
\end{tabular}

Abstract AB0955 Table 2. treatment during paediatric follow-up

\begin{tabular}{lc}
\hline TERAPIA & $\mathbf{N}(\%)$ \\
\hline Intrarticular infiltrations & $13(65 \%)$ \\
Systemic Steroids & $11(55 \%)$ \\
Methotrexate & $15(75 \%)$ \\
Hydroxychloroquine & $1(5 \%)$ \\
Leflunomide & $1(5 \%)$ \\
Sulfasalazine & $4(20 \%)$ \\
Cyclosporin A & $2(10 \%)$ \\
Biologic DMARDs & $11(55 \%)$ \\
\hline
\end{tabular}

Disclosure of Interests: Francesca Crisafulli: None declared, Marco Cattalini: None declared, Francesca Ricci: None declared, Giada Maffeis: None declared, Franco Franceschini: None declared, Angela Tincani Consultant for: UCB, Pfizer, Abbvie, BMS, Sanofi, Roche, GSK, AlphaSigma, Lillly, Jannsen, Cellgene, Novartis, Micol Frassi: None declared DOI: 10.1136/annrheumdis-2019-eular.7298

\section{AB0956 JIA ASSOCIATED TO CHROMOSOME 18 SHORT ARM DELETION: A CASE REPORT}

Rawane Dagher ${ }^{1}$, Marie Samarani ${ }^{1}$, Fouad Minkara ${ }^{2}$, Marie Claude Fadous Khalife ${ }^{1}{ }^{1}$ Notre Dame Des Secours University Hospital, Pediatrics, Byblos, Lebanon; ${ }^{2}$ Monla Hospital, Rheumatology, Tripoli, Lebanon

Background: Monosomy $18 p$ is a rare chromosomal disease resulting from complete or partial deletion of chromosome 18 short arm. It is associated with a variable phenotypic spectrum. Most reported patients have holoprosencephaly, developmental delay, short stature and distinctive facial features while autoimmune diseases are rarely seen. Manifestations of this syndrome vary depending on the extent of deletions.

Objectives: We report the case of a child with juvenile arthritis and uveitis associated to $18 p$ deletion syndrome.

Methods: A 5-year-old girl diagnosed with 18p deletion presented for pain and swollen joints since more than a year. 
She is the first born of a 51-year-old father and 40-year-old mother, nonconsanguineous parents, at full term, and has a history of early hypotonia, psychomotor developmental delay and failure to thrive.

Physical exam revealed arthritis of both ankles, the right knee and the MCP joint of the left thumb. We noted loose joints, high-arched palate and facial dysmorphism (hypertelorism, large protruding ears, flat nose and short philtrum). Body weight and height were at -3SD. Intellectual disability was mild.

Laboratory tests showed slightly increased ESR (35mm at first hour) with positive ANA (>1/1000) and negative RF and anti-CCP. Karyotype is 46XX with deletion of the short arm of chromosome 18. Both parents had normal Karyotypes. MRI of the brain showed cortical atrophy and periventricular leucomalacia.

Ophthalmic screening detected bilateral anterior uveitis.

The patient was treated by AINS and methotrexate along with topical ophthalmic steroids. 6 months after treatment, the child had complete regression of uveitis and arthritis.

Results: This case of de-novo $18 p$ deletion is associated to oligoarticular JIA with uveitis. According to our knowledge, there have only been 2 published cases of juvenile inflammatory arthritis with $18 \mathrm{p}$ deletion although 5 other cases were reported in association to other chromosome 18 abnormalities (deletion of long arm and ring). Few reports of adultonset arthritis with monosomy $18 p$ are available.

The exact explanation of this association is still unclear. As gene loss is a major factor in the pathogenesis of inflammation, this presentation might be related to deletion of PTPN2, a gene that has been associated to rheumatoid arthritis. PTPN2 encodes Protein Tyrosine Phosphatase Non-receptor type 2 and is located in the short arm of chromosome 18 PTPN2-deficiency stimulates $T$ follicular helper cell and B cell responses thus promoting autoimmune reaction. Of the 67 genes in $18 p$, only around 12 (of which PTPN-2) are responsible of a phenotype when hemizygous. Further genetic studies are needed to better elucidate the role of deleted genes in the pathogenesis of inflammation in monosomy $18 \mathrm{p}$. Conclusion: In addition to skeletal deformities such as kyphosis and feet deformities, children with $18 p$ deletion should be screened for inflammatory arthritis.

\section{REFERENCES}

[1] M.Hasi-Zogaj,C.Sebold,P. Heard,E.Carter,B.Soileau,D. Rupert and al. A review of $18 p$ deletions. American journal of medical genetics. Part $\mathrm{C}$, seminars in medical genetics. 2015 Sep;169(3):251-64.

[2] A.Chau, KH. Ramesh,AD. Jagannath, S. Arora. Rheumatoid arthritis in an adult patient with mosaic distal 18q-, 18p- and ring chromosome 18.F1000 research. 2018;6:1940.

[3] C.Ciccacci, P.Conigliaro,C.Perricone,S.Rufini,P.Triggianese and al. Polymorphisms in STAT-4, IL-10, PSORS1C1, PTPN2 and MIR146 Agenes are associated differently with prognostic factors in Italian patients affected by rheumatoid arthritis. Clinical and experimental immunology.2016:186:157-163

[4] F.Wiede,F.Sacirbegovic,Y.A.Leong,D.Yu,T.Tiganis. PTPN2 deficiency exacerbates $T$ follicular helper and $B$ cell responses and promotes the development of autoimmunity. The journal of immunology.2017;76:85-100.

Disclosure of Interests: None declared

DOI: 10.1136/annrheumdis-2019-eular.3774

\section{\begin{tabular}{l|l} 
AB0957 & ATYPICAL PRESENTATION AND EARLY RECURRENCE
\end{tabular} OF KAWASAKI DISEASE IN A FEMALE INFANT: CASE REPORT}

Rodrigo del Toro Rojas, Patricio Javier Flores Lopez, Luis Fernando Sanchez Espino, Cesar Adrian Martinez Longoria, Hector Enrique Valdes Garza. Instituto Tecnológico y de Estudios Superiores de Monterrey, TecSalud Pediatría, San Pedro Garza García, Mexico

Background: Kawasaki Disease (KD) is the second most common vasculitis in the pediatric population and the leading cause for pediatric acquired heart disease in developed countries. It is commonly diagnosed in the Mexican pediatric population, epidemiology in this country is not established, since cases are not usually reported to the healthcare system. The clinical features are quite variable, but diagnosis and prompt treatment will decrease morbidity and mortality. Coronary artery aneurysms are the most common complication, which represent the leading cause of acute coronary syndrome before 40 years of age. Recurrence of KD is estimated to be around $3 \%$ in Japanese patients and $1 \%$ in the United States, nevertheless, this data in Latin-American children is unknown. It usually affects patients before reaching 3 years old and within 2 years of the initial attack, presenting with an abrupt onset and higher complication rates, requiring aggressive workup, treatment and follow-up.

Objectives: To review an atypical presentation of $\mathrm{KD}$ and early recurrence in a 7-month-old female.

Methods: We present a 7-month-old female diagnosed with KD at 48 days old. She presented to the emergency unit with irritability, high and persistent fever and acholia. During workup, she was found with cholestasis and gallbladder hydrops. Negative CSF, blood and urine cultures were documented during hospitalization, and fulfilled the KD criteria. The cardiac ultrasound revealed coronary abnormalities: a RCA of $2.8 \mathrm{~mm}$ (Z Score +4.79), LCA of $3.3 \mathrm{~mm}$ (Z-Score +5.69) and LAD of $2.4 \mathrm{~mm}$ (Z SCORE +4.25), which fit into classification 3 and 4 , as small and medium aneurysms, according to AHA 2017. Immunoglobulin $(2 \mathrm{~g} / \mathrm{kg})$ and aspirin $(80 \mathrm{mg} / \mathrm{kg})$ were administered and she was discharged 36 hours after the IVIg infusion, afebrile and with ambulatory follow-up, 30 days later the coronary abnormalities showed RCA of $2.0 \mathrm{~mm}$ (Z-Score +2.3 ) LCA of $3.0 \mathrm{~mm}$ (Z-Score +6), LAD $2.3 \mathrm{~mm}$ (Z-Score +2.9). Six months later, she presented fever for 6 days, irritability and polymorphous rash. On physical exam BCGitis, non-suppurative conjunctivitis and pallor in hands and feet, elevated CRP and ESR, leukocytosis, thrombocytosis and sterile leukocyturia. Echocardiography reported RCA od $2.4 \mathrm{~mm}$ (Z Score +4.8), LCA of 3.1 (Z-Score +4.2) and LAD of $2.3 \mathrm{~mm}$ (Z-Score +3.2 ), diagnosing KD recurrence, admitting the patient for IVIg and aspirin administration.

Results: The patient was treated with IVIg and aspirin. Follow-up by Cardiology determined improvement of Z-Scores. Recurrence occurred with worsening of the cardiac abnormalities. Cardiac prognosis is importantly affected due to the atypical age, vascular abnormalities and repeated vasculitic process. Rheumatologic consult should be considered since disease like Takayasu Arteritis, Polyarteritis Nosa and ADA2 deficiency need to be ruled out.

Conclusion: KD needs prompt diagnosis and treatment due to the poten tial consequences when delayed. Clinical suspicion is important due to the possible atypical presentation. As with this patient, age, gender and presentation are not exclusive. Despite adequate treatment, recurrence and worsening of the cardiac abnormalities occurred. Both KD events before 1 year old and with atypical presentations. Rheumatologic and cardiac follow-up need to be stringent through lifetime, to determine pharmacologic treatment, as ell as physical activity and reproductive counseling

\section{REFERENCES}

[1] McCrindle BW, Rowley AH, Newburger JW, Burns JC, Bolger AF, Gewitz $M$, et al. Diagnosis, Treatment and Long-term Management of Kawasaki Disease: A scientific Statement for Health Professionals From the American Heart Association. Circulation 2017;135(17):e927-e99.

Disclosure of Interests: None declared

DOI: 10.1136/annrheumdis-2019-eular.6490

\section{AB0958 $\quad$ PEDIATRIC BEHCET'S DISEASE WITH SINUS VENOUS THROMBOSIS: THREE CENTER EXPERIENCE FROM} TURKEY

Selcan Demir ${ }^{1}$, Ceyhun Acarı ${ }^{2}$, Özge Basaran ${ }^{3}$, Erdal Sag ${ }^{1}$, Kader Karlı Oğuz ${ }^{4}$, Yelda Bilginer ${ }^{1}$, Erbil Unsal' ${ }^{2}$, Seza Özen ${ }^{1} .{ }^{1}$ Hacettepe University Faculty of Medicine, Pediatric Rheumatology, Ankara, Turkey; ${ }^{2}$ Dokuz Eylül University Faculty of Medicine, Pediatric Rheumatology, Ankara, Turkey; ${ }^{3}$ Ankara Child Health and Disease Hematology Oncology Training and Research Hospital, University of Health Sciences, Pediatric Rheumatology, Ankara, Turkey; ${ }^{4}$ Hacettepe University Faculty of Medicine, Radiology, Ankara, Turkey

Background: Behçet's disease (BD) is an autoinflammatory disorder which may present with multi-systemic involvements including the vascular, cutaneous, articular, gastrointestinal, and/or central nervous systems (CNS). CNS involvement appears in two main groups: parenchymal or non-parenchymal. Neurological symptoms in children and adolescents can be confused with many other disorders and may be the initial symptom of BD. Objectives: To report our experiences of the juvenile Behçet's Disease patients with cerebral venous sinus thrombosis (CVST) and to review previous studies reporting the clinical characteristics and outcomes of Juve nile Behçet's Disease with CVST.

Methods: The patients who met Pediatric Behçet's Disease (PEDBD) classification criteria for juvenile Behçet's Disease from 3 referral centers in Turkey were reviewed retrospectively. Disease activity was assessed by $\mathrm{BD}$ current activity form (BDCAF). A systematic review of literature of all published data was conducted. 